\title{
Electronic structure of the negatively charged silicon-vacancy center in diamond
}

\author{
Lachlan J. Rogers, $,{ }^{1,}, \dagger$ Kay D. Jahnke, ${ }^{1, \dagger}$ Marcus W. Doherty, ${ }^{2}$ Andreas Dietrich, ${ }^{1}$ Liam P. McGuinness, ${ }^{1}$ Christoph Müller, ${ }^{1}$ \\ Tokuyuki Teraji, ${ }^{3}$ Hitoshi Sumiya, ${ }^{4}$ Junichi Isoya, ${ }^{5}$ Neil B. Manson, ${ }^{2}$ and Fedor Jelezko ${ }^{1}$ \\ ${ }^{1}$ Institut für Quantenoptik and IQST, Universität Ulm, D-89081 Ulm, Germany \\ ${ }^{2}$ Laser Physics Centre, Research School of Physics and Engineering, Australian National University, ACT 0200, Australia \\ ${ }^{3}$ National Institute for Materials Science, 1-1 Namiki, Tsukuba, Ibaraki 305-0044, Japan \\ ${ }^{4}$ Advanced Materials $R$ \& D Laboratories, Sumitomo Electric Industries Ltd, Itami, Hyogo 664-0016, Japan \\ ${ }^{5}$ Research Center for Knowledge Communities, University of Tsukuba, 1-2 Kasuga, Tsukuba, Ibaraki 305-8550, Japan
}

(Received 10 October 2013; revised manuscript received 18 March 2014; published 2 June 2014)

\begin{abstract}
The negatively charged silicon-vacancy $\left(\mathrm{SiV}^{-}\right)$center in diamond is a promising single-photon source for quantum communications and information processing. However, the center's implementation in such quantum technologies is hindered by contention surrounding its fundamental properties. Here we present optical polarization measurements of single centers in bulk diamond that resolve this state of contention and establish that the center has a $\langle 111\rangle$ aligned split-vacancy structure with $\mathrm{D}_{3 \mathrm{~d}}$ symmetry. Furthermore, we identify an additional electronic level and evidence for the presence of dynamic Jahn-Teller effects in the center's 738-nm optical resonance.
\end{abstract}

DOI: 10.1103/PhysRevB.89.235101

PACS number(s): 71.55.Cn, 61.72.jn, 81.05.ug

\section{INTRODUCTION}

Single quantum emitters in solids are promising sources of single photons [1,2], architectures for qubits [3], and biological probes [4-6]. The negatively charged nitrogen-vacancy $\left(\mathrm{NV}^{-}\right)$ color center in diamond is a prominent example [7], but its optical fluorescence is spread over a large phonon sideband with only $4 \%$ present in the zero phonon line (ZPL). In contrast, the negatively charged silicon-vacancy $\left(\mathrm{SiV}^{-}\right)$center in diamond is known to have a small phonon sideband, with $70 \%$ of its fluorescence concentrated in a ZPL at $738 \mathrm{~nm}$ $(1.68 \mathrm{eV})$ [8]. This is a marked advantage for applications that require indistinguishable photons, such as quantum communication and information processing architectures that rely on photon entanglement [9]. It is also beneficial for technological applications involving cavity QED [10-12]. However, persistent contention surrounding the fundamental properties of the $\mathrm{SiV}^{-}$center has hindered its implementation in such quantum technologies.

This contention arises mainly from conflicting reports of the geometrical alignment of $\mathrm{SiV}^{-}$within the diamond lattice. Optical polarization measurements have suggested the center is aligned along $\langle 110\rangle$ crystal vectors $[13,14]$, while a $\langle 111\rangle$ alignment was concluded from ab initio calculations [15] and related electron paramagnetic resonance (EPR) measurements of the neutral charge state $\left(\mathrm{SiV}^{0}\right)$ [16]. Further disagreement is added by a past observation of the Zeeman splitting of the 738-nm ZPL fine structure, which suggests that the center has a $\langle 100\rangle$ orientation [17].

\footnotetext{
*lachlan.rogers@uni-ulm.de

${ }^{\dagger}$ L.R. and K.J. contributed equally to this work.
}

Published by the American Physical Society under the terms of the Creative Commons Attribution 3.0 License. Further distribution of this work must maintain attribution to the author(s) and the published article's title, journal citation, and DOI.
Here we report a study of the polarization of photoluminescent emission (PL) and excitation (PLE) of single $\mathrm{SiV}^{-}$centers in bulk diamond at room and cryogenic temperatures, and confirm the $\langle 111\rangle$ alignment. Our study avoids the limitations of the previous polarization studies by utilizing single center interrogation in bulk samples which have well defined crystallographic orientation and low intrinsic strain. This alignment is consistent with a silicon split-vacancy structure having $D_{3 d}$ symmetry, and our conclusions are supported by recent Zeemann studies [18]. Additionally, by examining the polarization properties of the PL fine structure we have confirmed that the 738-nm ZPL arises from a ${ }^{2} \mathrm{E}_{\mathrm{g}} \leftrightarrow{ }^{2} \mathrm{E}_{\mathrm{u}}$ transition and that the fine structure arises from spin-orbit interactions. Analysis of the PL polarization of the accompanying phonon sideband strongly suggests dynamic Jahn-Teller effects that invite future investigation. Furthermore, our PLE polarization observations reveal an additional ${ }^{2} \mathrm{~A}$ electronic level, which is excited at wavelengths $\lesssim 605 \mathrm{~nm}(\gtrsim 2.05 \mathrm{eV})$. Based upon past PLE spectra [19] and the electronic model of the center [15,16], this level is precisely identified as ${ }^{2} \mathrm{~A}_{1 \mathrm{~g}}$. These observations provide the most complete understanding of the $\mathrm{SiV}^{-}$electronic structure yet obtained.

\section{POLARIZATION OF PHOTOLUMINESCENCE}

Two samples with $\mathrm{SiV}^{-}$densities of $\sim 0.1 \mu \mathrm{m}^{-3}$ were used in our experiments: one with a $\{111\}$ face and one with a $\{100\}$ face. The first sample was a low strain high-pressure high-temperature (HPHT) diamond that was laser cut to provide the desired $\{111\}$ surface. The $\mathrm{SiV}^{-}$sites found in this surface were naturally occurring and must have been formed during the HPHT growth process. The $\{100\}$ sample was a microwave plasma-assisted chemical-vapor-deposition (MPCVD) film grown on a $\langle 100\rangle$-oriented plate cut from a lowstrain, type-IIa, HPHT crystal (Sumitomo Electric Industries, Ltd.). Details of the MPCVD apparatus have been described elsewhere [20]. Si atoms were introduced into the growth plasma as it etched a $6 \mathrm{H}-\mathrm{SiC}$ single-crystal plate which was placed in the sample chamber. $\mathrm{SiC}$ has a greater resistance 


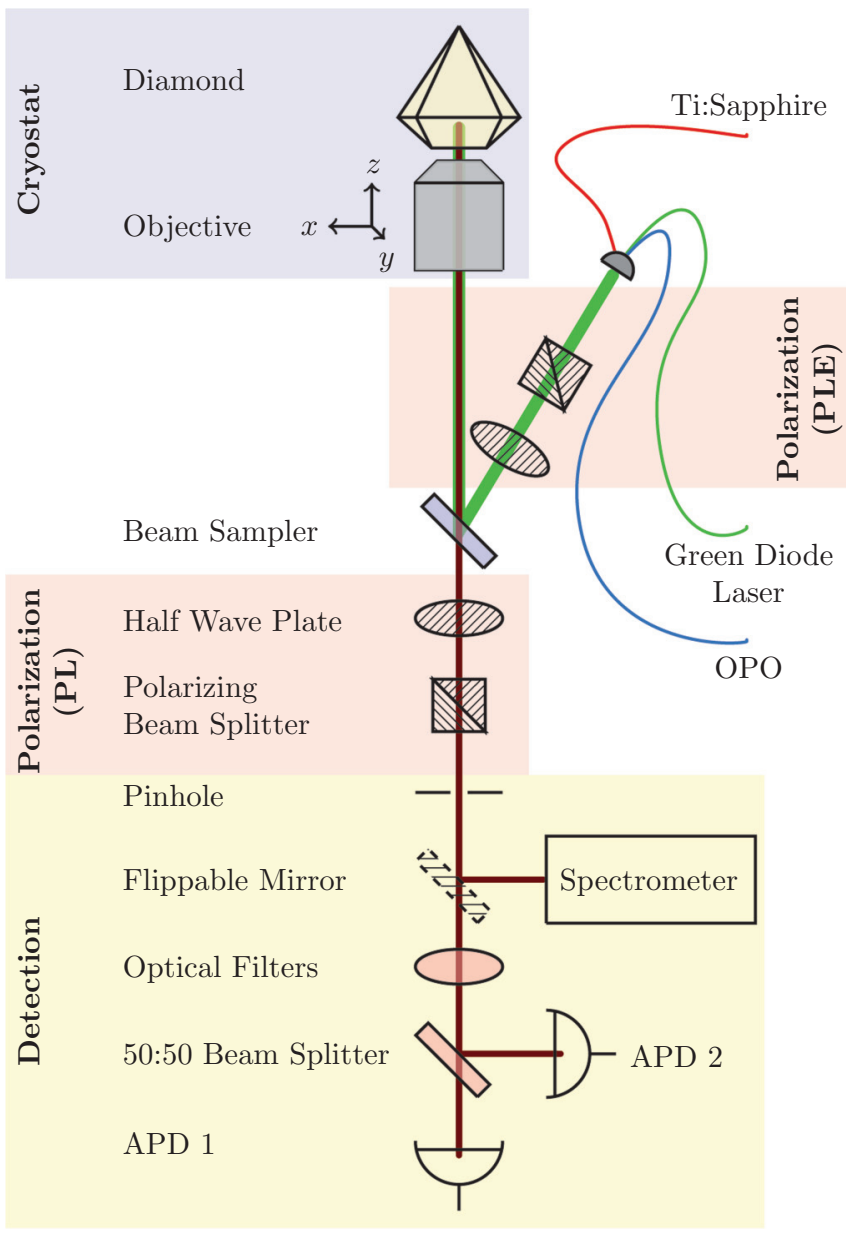

FIG. 1. (Color online) Experimental setup. The custom-built confocal microscope was built around a continuous flow helium cryostat that held the sample. A 532-nm green diode laser was used for excitation in the PL measurements, and a half wave plate and polarizer allowed the fluorescence to be measured as a function of polarization angle. The $\mathrm{SiV}^{-}$fluorescence (dark red) was then measured either using avalanche photodiodes (APDs) or with a spectrometer. PLE measurements were performed using a $\mathrm{cw}$ titanium sapphire laser and a pulsed optical parametric oscillator (OPO). In order to measure the polarization dependence of the excitation transition, the HWP and polarizer were moved from the fluorescence beam to the laser beam at the fiber outcoupler.

to hydrogen plasma than both $\mathrm{Si}$ and $\mathrm{SiO}_{2}$, which slowed the $\mathrm{Si}$ incorporation rate enough to produce $\mathrm{SiV}^{-}$centers with a density that allowed individual sites to be identified.

A green diode laser at $532 \mathrm{~nm}$ was used to excite $\mathrm{SiV}^{-}$for the photoluminescence (PL) measurements. The fluorescence was measured using either a pair of avalanche photodiodes (APDs) or a spectrometer with a 1596 grooves $/ \mathrm{mm}$ grating. The samples were mounted on the cold finger of a continuous flow helium cryostat capable of cooling them to about $8 \mathrm{~K}$. A custom built confocal microscope (illustrated in Fig. 1) was used to study individual color centers, which were confirmed to be single sites using second-order autocorrelation measurements. Excitation power was of order $1 \mathrm{~mW}$ at the microscope objective. A half wave plate (HWP) and a linear polarizer were placed in the detection beam so that rotating the HWP allowed fluorescence to be measured at arbitrary polarization angles. Having the linear polarizer fixed and rotating the fluorescence beam with the HWP eliminated the influence of any polarization dependence of the measurement apparatus.

\section{A. $\{111\}$ surface}

More than 40 fluorescent sites in the $\{111\}$-faced sample were examined using spectrometer measurements. Over half of these sites were found to be either $\mathrm{NV}^{-}$or a $\mathrm{SiV}^{-}$close to a $\mathrm{NV}^{-}$, but 15 pure $\mathrm{SiV}^{-}$sites were observed. Seven typical $\mathrm{SiV}^{-}$sites lay within a convenient scan region, and these were measured in further detail. In particular, the spectrally resolved ZPLintensity was measured at $8 \mathrm{~K}$ as the detection polarization was rotated. The resulting ZPL polarizations formed distinct sets as shown in Fig. 2(a).

We discuss the polarization dependence in terms of polarization contrast

$$
C=\frac{I_{\max }-I_{\min }}{I_{\max }+I_{\min }}
$$

given as a percentage $[14,21]$. For the $\{111\}$ surface there was a set of sites (number (1) which showed no polarization dependence $(C=3 \%)$. Two other sets (numbered (2) and (3)) each showed a contrast of $C=60 \%$ and had their maxima separated by $120^{\circ}$.

These sets correspond to the pattern of $\langle 111\rangle$ vectors in the diamond lattice when viewed through a $\{111\}$ surface, as illustrated in Fig. 2(b). The $\mathrm{NV}^{-}$sites also formed sets corresponding to the $\langle 111\rangle$ vectors. However, none of the $40 \mathrm{NV}^{-}$and $\mathrm{SiV}^{-}$sites were observed in orientation (4), which was the growth direction of this crystal sector. There are numerous reports of preferential orientation when defect sites are incorporated during growth [16,22-26]. The absence of orientation (4) is attributed to similar growth effects, and it does not impact the strength of our subsequent conclusions. The exact correlation of the pattern of $\mathrm{SiV}^{-}$sites with the pattern of $\langle 111\rangle$ crystal vectors is strong evidence that $\mathrm{SiV}^{-}$is aligned along these directions in the lattice. (a)

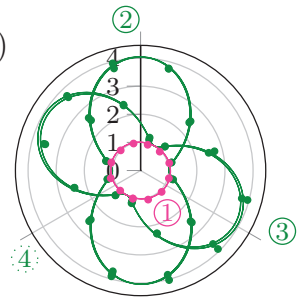

(b)

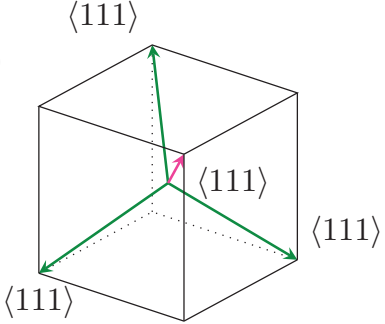

FIG. 2. (Color online) Emission ZPL intensity as a function of

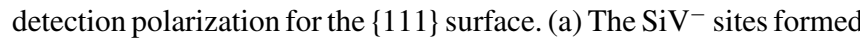
distinct sets, within which all sites had similarly polarized emission. One set had no polarization variation $(C=3 \%)$, while two other sets had a contrast of $C=60 \%$ and were separated by $120^{\circ}$. (b) These sets are arranged in a manner corresponding to the projection of the $\langle 111\rangle$ crystal vectors, indicating that the $\mathrm{SiV}^{-}$center has a $\langle 111\rangle$ major symmetry axis. The position of an expected fourth set that should share the contrast of $C=60 \%$ is marked, and the absence of any $\mathrm{SiV}^{-}$in this orientation is attributed to crystal-growth effects. 
(a)

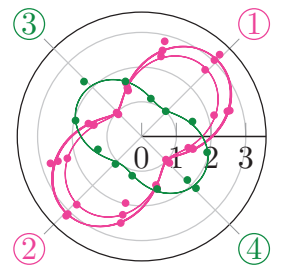

(b)

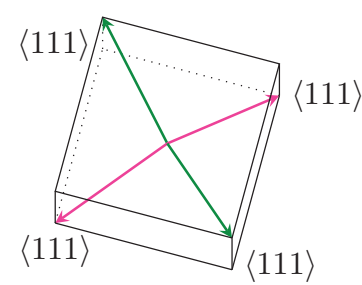

FIG. 3. (Color online) Emission ZPL intensity as a function of detection polarization for the $\{100\}$ surface. (a) $\mathrm{The}_{\mathrm{SiV}}^{-}$sites formed two distinct sets with contrast of about $C=50 \%$, separated by $90^{\circ}$. (b) These sets are also arranged in a manner corresponding to the projection of the $\langle 111\rangle$ crystal vectors. The difference in contrast between the perpendicular sets is attributed to the viewing axis being slightly misaligned from $\langle 100\rangle$, as is the case in the illustrated unit cube.

\section{B. $\{100\}$ surface}

The same measurement was made for eight $\mathrm{SiV}^{-}$sites in the $\{100\}$ sample, resulting in two distinct sets of sites separated by $90^{\circ}$ as shown in Fig. 3(a). As before, these are consistent with the pattern of $\langle 111\rangle$ vectors in the viewing projection as illustrated. Note that since the patterns are perpendicular and the $\langle 111\rangle$ vectors form perpendicular sets, it is also possible to interpret the patterns as implying that the polarization is orthogonal to $\langle 111\rangle$ (instead of along it). This ambiguity arises fundamentally from the geometry, and hinders the $\{100\}$ surface from clearly revealing the $\mathrm{SiV}^{-}$dipole properties. For

this reason we focus now on the $\{111\}$ surface for fine-structure measurements.

\section{ZPL fine structure}

Polarization measurements probe the dipole moments that are active in a given transition. In general, an emitter can be fully characterized by considering three orthogonal dipoles. The conventional site-referenced axes for a defect aligned to

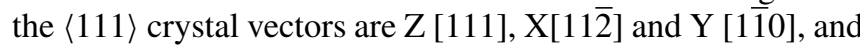
so we will consider dipole moments along these local axes. A dipole produces light polarized parallel to its axis, but this light radiates primarily into the plane orthogonal to its axis [Fig. 4(a)]. In the $\{111\}$ surface, $\mathrm{SiV}^{-}$orientation (1) has its Z axis aligned to the viewing direction (Fig. 2). Since a dipole does not radiate along its axis, the measured fluorescence can only arise from the $\mathrm{X}$ and $\mathrm{Y}$ dipole moments. The lack of contrast indicates that the $\mathrm{X}$ and $\mathrm{Y}$ dipole moments have equal magnitude, denoted $d_{\perp}$. The axial dipole moment (along Z) is called $d_{\|}$

The other three $\mathrm{SiV}$ orientations in the $\{111\}$ sample have their $\mathrm{Z}$ axes inclined at $19.5^{\circ}$ to the surface. For these three oblique orientations there is always a choice of $\mathrm{X}$ and $\mathrm{Y}$ that makes $\mathrm{Y}$ perpendicular to the surface. The corresponding $\mathrm{X}$ then always appears parallel to Z, as illustrated in Fig. 4(c). To examine the obliquely oriented sites with clarity we take $\mathrm{X}, \mathrm{Y}, \mathrm{Z}$ as conventionally defined, and choose the sample surface to be $S=(11 \overline{1})$. The scalar product allows a quick determination of the angles that $\mathrm{X}, \mathrm{Y}, \mathrm{Z}$ form with the surface $S$, making it possible to obtain the perceived strength of dipole (a)
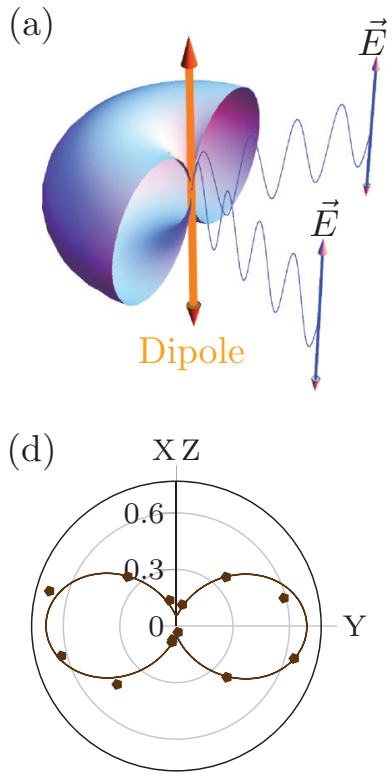

(b)

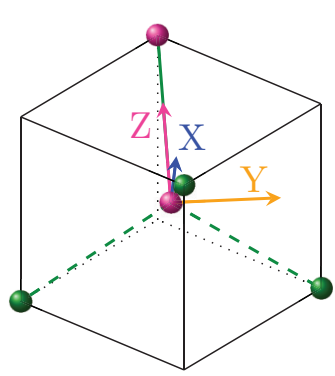

(e)

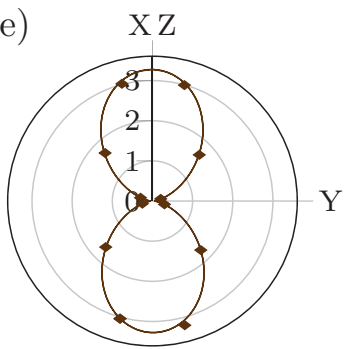

(c)
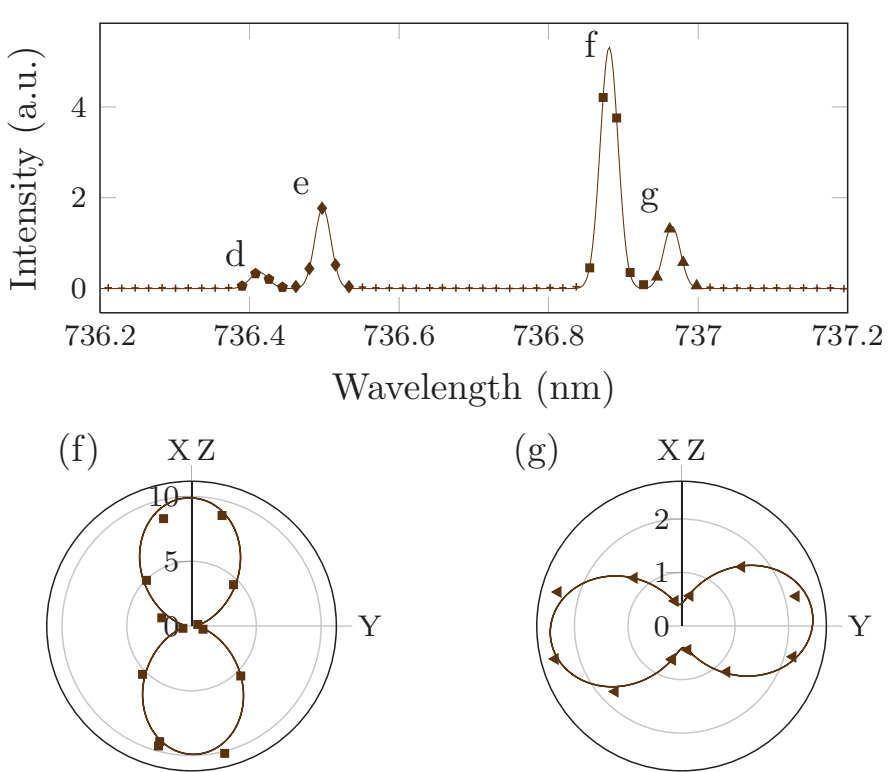

$(\mathrm{g})$

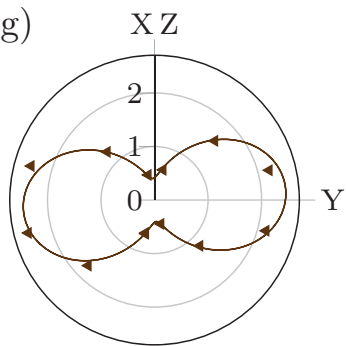

FIG. 4. (Color online) Polarization of ZPL fine structure. (a) A dipole radiates primarily into the plane orthogonal to its axis. The toroidal surface illustrates the relative intensity as a function of elevation angle out of this plane, and importantly shows that no radiation propagates along the dipole axis. In every direction of propagation, the radiation is polarized parallel to the dipole axis (here E indicates the electric-field

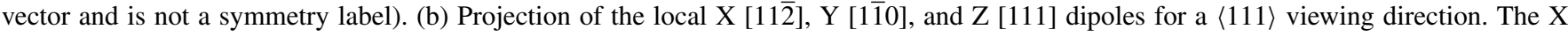
axis lies in a symmetry plane, and so each of the three oblique $\mathrm{SiV}^{-}$orientations may have $\mathrm{Y}$ chosen so that it is perpendicular to the viewing direction. The projection of $\mathrm{X}$ is parallel to that of $\mathrm{Z}$. (c) At $8 \mathrm{~K}$ the ZPL is resolved into four components. The width of these lines is limited by the spectrometer resolution. (d)- $(\mathrm{g})$ The polarization of each ZPL component. The two inner lines are polarized in the XZ direction, and have almost complete contrast $\left(C_{(e)}=97 \%\right.$ and $\left.C_{(f)}=92 \%\right)$. The two outer lines are oppositely polarized $(\mathrm{Y})$, and have contrasts $C_{(d)}=85 \%$ and $C_{(g)}=72 \%$. 
moments along these axes. Because $\mathrm{Y}$ is perpendicular to the viewing direction the dipole moment in this axis is seen at $100 \%$. Due to the geometry, only $33 \%$ of the dipole moment along $\mathrm{X}$ and $94 \%$ of that along $\mathrm{Z}$ are observed. Thus the expected polarization contrast for a transition only involving $d_{\perp}$ (i.e., $\left.d_{\|}=0\right)$ is

$$
C_{d_{\perp}}=\frac{1-0.33}{1+0.33}=50 \%
$$

with maxima in the Y direction. A transition for which $d_{\perp}=0$ should have contrast of $C_{d_{\|}}=100 \%$ with maxima in the $\mathrm{XZ}$ direction (but only $94 \%$ of the intensity will be observed).

At $8 \mathrm{~K}$ the ZPL was spectrally resolved into four components as shown in Fig. 4(a). Photoluminescence from each of the four ZPL components was measured at various polarizations, and the results are shown against the projected $\mathrm{XZ}$ and $\mathrm{Y}$ axes in Figs. 4(d)-4(g). The inner two lines are polarized along XZ and have a contrast of $C \geqslant 92 \%$, while the outer lines are polarized in the $\mathrm{Y}$ direction with contrasts of $C=72 \%$ for (d) and $C=85 \%$ for $(\mathrm{g})$.

Since the inner two lines have nearly complete contrast and are polarized in the $\mathrm{XZ}$ direction they must arise only from the axial dipole moment $d_{\|}$. The outer two lines are polarized in the Y direction, but have contrast greater than the $50 \%$ expected for a purely $d_{\perp}$ transition. For optical detection normal to the surface, this suggests that the $\mathrm{Y}$ dipole moment is stronger than that along $\mathrm{X}$ which is in conflict with the observations for orientation (1). However, the microscope objective used here collects fluorescence within a solid angle around the surface normal. This amplifies the apparent strength of the Y dipole moment and leads to higher than expected contrast. This effect was assessed by measuring nearby $\mathrm{NV}^{-}$centers, which are also $\langle 111\rangle$ aligned but have only a perpendicular dipole moment. For $\mathrm{NV}^{-}$sites obliquely angled to the surface, polarization contrast was found to be $\sim 70 \%$ indicating that $\mathrm{SiV}^{-}$line $(\mathrm{g})$ in Fig. 4 arises purely from $d_{\perp}$. Line (d) is similar, but as it has the weakest intensity (and so highest uncertainty in contrast), it is difficult to draw detailed conclusions.

The magnitudes of the parallel and transverse dipole moments may also be compared. It is clear from Fig. 4 that the inner two lines, arising from $d_{\|}$, are stronger than the outer lines which arise from $d_{\perp}$. The difference in intensity indicates that $d_{\|}$is about four times stronger than $d_{\perp}$. This indicates that the ZPL is predominately polarized parallel to $\langle 111\rangle$, allowing the $\mathrm{SiV}$ center to be approximated as a single dipole.

Applying symmetry selection rules from group theory, a transition with differing $\mathrm{Z}$ and equal $\mathrm{X}$ and $\mathrm{Y}$ dipoles at a $\langle 111\rangle$ aligned site in diamond can only occur if the site has $\mathrm{C}_{3 \mathrm{v}}$ or $\mathrm{D}_{3 \mathrm{~d}}$ symmetry and if the transition occurs between two E electronic levels [27]. This conclusion is consistent with previous interpretations of the four-line fine structure $[15,28,29]$. A splitting of the degeneracy in each $\mathrm{E}$ state produces four transitions as shown in Fig. 5. We observed that the ground- and excited-state splittings are the same for each of the single centers we studied and are 0.20 and $1.05 \mathrm{meV}$, respectively. This indicates that these splittings arise from intrinsic properties of $\mathrm{SiV}^{-}$.

Based upon our polarization measurements, in Fig. 5(a) we assign the dipole selection rules of the fine-structure transitions between the E electronic levels. The observed

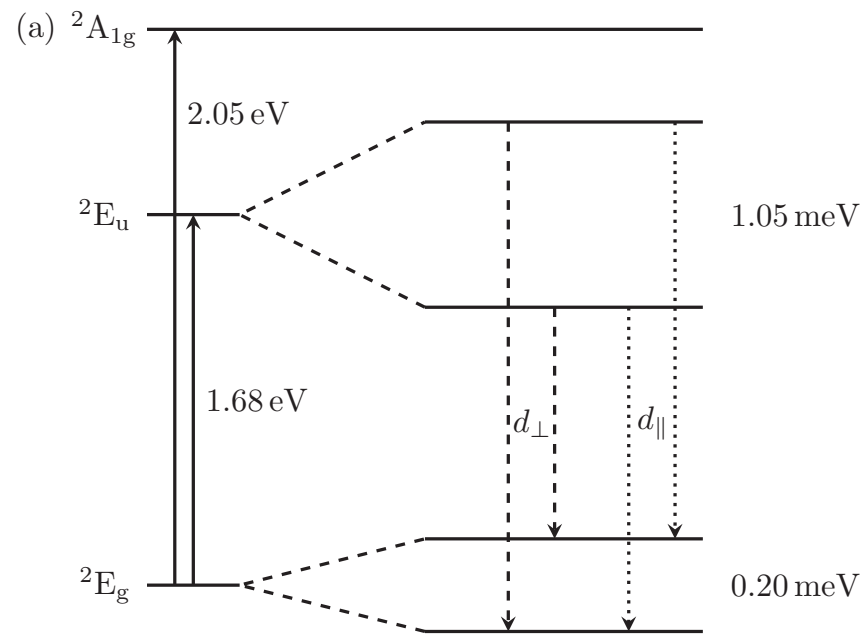

(b)

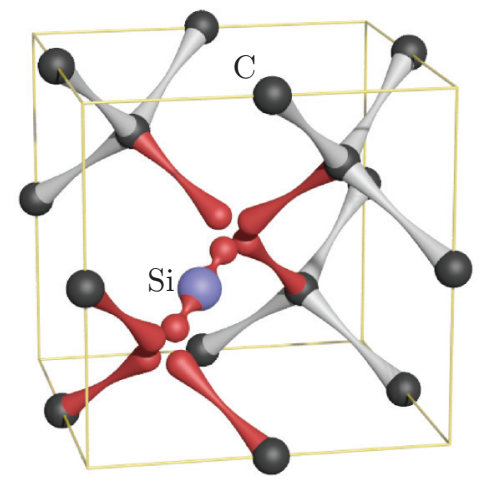

FIG. 5. (Color online) (a) Electronic structure of $\mathrm{SiV}^{-}$, depicting the ground ${ }^{2} \mathrm{E}_{\mathrm{g}}$ and first excited ${ }^{2} \mathrm{E}_{\mathrm{u}}$ levels [15], their doublet fine structure [29], and the ${ }^{2} \mathrm{~A}_{1 \mathrm{~g}}$ level identified in this work. The ${ }^{2} \mathrm{E}_{\mathrm{g}} \leftrightarrow{ }^{2} \mathrm{E}_{\mathrm{u}}$ transition gives rise to the four-line fine structure of the $738-\mathrm{nm}$ $(1.68 \mathrm{eV})$ ZPL. The depicted polarization selection rules are as observed and correspond to a spin-orbit origin of the fine structure. Comparison with past PLE spectra [19] suggests that the ${ }^{2} \mathrm{~A}_{1 \mathrm{~g}}$ level is $605 \mathrm{~nm}(2.05 \mathrm{eV})$ above the ground state. (b) Schematic of the split-vacancy structure with $\mathrm{D}_{3 \mathrm{~d}}$ symmetry. The "dangling bonds" contributing to the $\mathrm{SiV}^{-}$center are highlighted in red. These are drawn schematically to indicate the lattice vacancies, and do not correspond to actual electron charge distributions.

selection rules are consistent with a spin-orbit origin of the zero-field splittings. Within each ${ }^{2}$ E level, the interaction of the electronic $S=1 / 2$ spin and orbital angular momentum about the $\langle 111\rangle$ symmetry axis leads to a splitting between sublevels where the angular momenta constructively and destructively combine.

The fine-structure polarization measurements are not able to distinguish between $\mathrm{C}_{3 \mathrm{v}}$ and $\mathrm{D}_{3 \mathrm{~d}}$ site symmetries. The precise symmetry of the center may only be established through evidence for the absence $\left(\mathrm{C}_{3 \mathrm{v}}\right)$ or presence $\left(\mathrm{D}_{3 \mathrm{~d}}\right)$ of inversion symmetry [27]. However, ab initio calculations have suggested the $\mathrm{D}_{3 \mathrm{~d}}$ symmetry for $\mathrm{SiV}^{-}$[15]. This model places the $\mathrm{Si}$ 

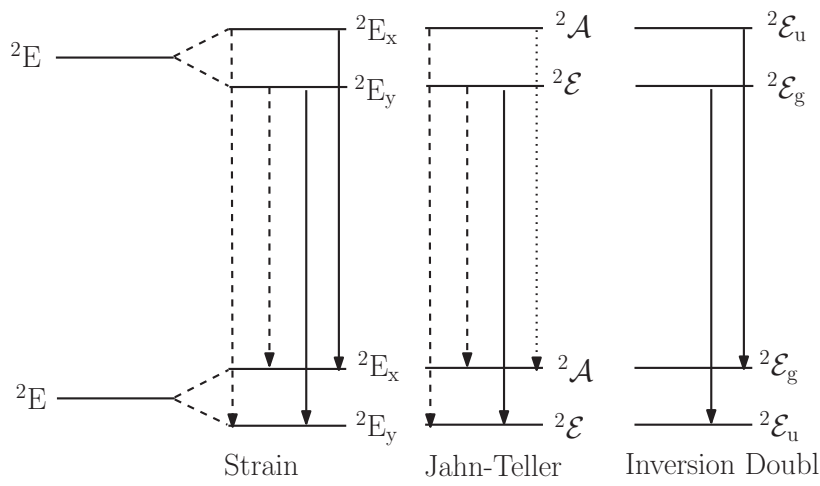

Inversion Doubling

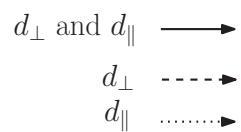

FIG. 6. The polarization selection rules of the alternate origins of the optical ZPL fine structure that are inconsistent with experiment: strain, the Jahn-Teller effect, and inversion doubling. Strain lowers the symmetry of the center and splits both the orbitally degenerate ground and excited ${ }^{2} \mathrm{E}$ electronic levels into individual orbital levels denoted $E_{x}$ and $E_{y}$. The Jahn-Teller effect couples the ${ }^{2} E$ electronic levels with E vibrational modes to yield lowest ${ }^{2} \mathcal{E}$ and ${ }^{2} \mathcal{A}$ vibronic levels [30]. Note that the relative intensities of the fine-structure lines depends on the Jahn-Teller energy. In inversion doubling at a $\mathrm{D}_{3 \mathrm{~d}}$ site, the ${ }^{2} E_{g}$ and ${ }^{2} E_{u}$ electronic levels are coupled with an $A_{u}$ vibrational mode to yield odd ${ }^{2} \mathcal{E}_{u}$ and even ${ }^{2} \mathcal{E}_{g}$ vibronic levels [35].

atom in the middle of two adjacent vacancies, as illustrated in Fig. 5(b).

Importantly, the observed selection rules are inconsistent with a strain origin of the splittings (as illustrated in Fig. 6). The negligible effect of strain is further supported by the observed homogeneity of splittings in our sample. Figure 6 also shows that our observed selection rules are inconsistent with the other two previously proposed origins of the fine structure: the static Jahn-Teller effect [15], and inversion doubling at a site with $\mathrm{D}_{3 \mathrm{~d}}$ symmetry [29].

\section{EXCITATION POLARIZATION INDICATES A NEW ELECTRONIC TRANSITION}

Despite its weak emission phonon sideband that extends only $100 \mathrm{~nm}(200 \mathrm{meV})$, PL of the 738-nm ZPL is easily excited using 532-nm light $(450 \mathrm{meV}$ above the extent of the absorption band). In order to investigate this unexpected phenomena, we measured the PLE polarization dependence at $532 \mathrm{~nm}$. To perform these experiments the HWP and polarizer were moved from the detection path to the incoming laser beam as illustrated in Fig. 1. Figure 7(a) shows the polarization contrast for the three orientations seen in the $\{111\}$ face. While the contrast seems similar to the PL measurements in Fig. 2, the oblique orientations (2) and (3) have their PLE polarized opposite to their PL. Instead of having maxima along the XZ direction, the PLE measurements show maxima in the $\mathrm{Y}$ direction. Combined with the contrast of about $70 \%$, this indicates that the excitation transition only involves $d_{\perp}$ (as discussed in the previous section).

For a $\langle 111\rangle$ aligned site with $\mathrm{C}_{3 \mathrm{v}}$ or $\mathrm{D}_{3 \mathrm{~d}}$ symmetry, these dipole selection rules correspond to a transition between $\mathrm{E}$ and $\mathrm{A}$ electronic levels and imply the presence of a ${ }^{2} \mathrm{~A}$ level. Theoretical calculations of the $\mathrm{D}_{3 \mathrm{~d}}$ split-vacancy model of the center predict such excited ${ }^{2} \mathrm{~A}_{1 \mathrm{~g}}$ and ${ }^{2} \mathrm{~A}_{2 \mathrm{u}}$ levels $[15,16]$. $\mathrm{A}{ }^{2} \mathrm{E} \rightarrow{ }^{2} \mathrm{~A}$ absorption from the ground ${ }^{2} \mathrm{E}$ level that is accompanied by a significant phonon sideband will explain the strong PLE observed at $532 \mathrm{~nm}$. The observed PL at $738 \mathrm{~nm}$ for 532-nm excitation will occur if the ${ }^{2} \mathrm{~A}$ level principally decays nonradiatively to the excited ${ }^{2} \mathrm{E}$ level.

In order to estimate the energy of the ${ }^{2} \mathrm{~A}$ level, the PLE polarization contrast was measured in the range $532-640 \mathrm{~nm}$ using a Ti:sapphire pumped optical parametric oscillator (OPO) and in the range 696-725 $\mathrm{nm}$ using a Ti:sapphire laser (a)

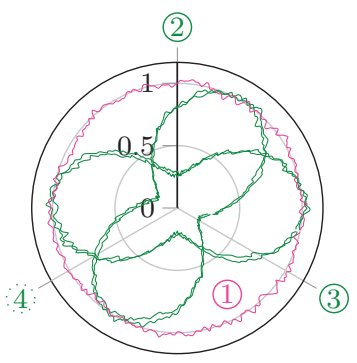

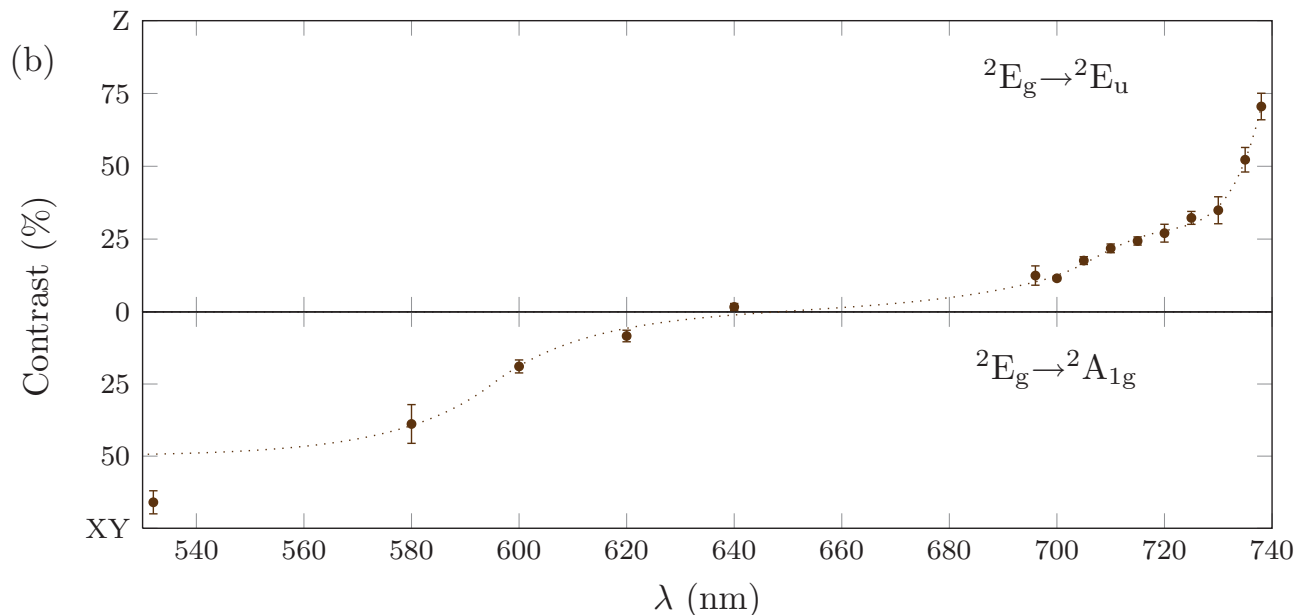

FIG. 7. (Color online) (a) The PLE polarization dependence for a 532-nm laser incident on the (111) surface. These have been normalized to polarization along Y and the PLE contrast $C_{\mathrm{PLE}}=66 \pm 4 \%$ is in the opposite sense to that in PL. (b) The absorption contrast over a range of laser wavelengths. The error bars indicate the statistical variation of polarization contrast measured over seven $\mathrm{SiV}^{-}$sites. The high contrast of the ZPL diminishes quickly for off-resonant excitation. The contrast changes to the "opposite" direction around $600 \mathrm{~nm}$, but the broad OPO line has smeared out this transition. The dotted line is a visual guide only. 
[see Fig. 7(b)]. The PLE contrast switches from corresponding to a ${ }^{2} \mathrm{E} \rightarrow{ }^{2} \mathrm{E}$ transition to $\mathrm{a}^{2} \mathrm{E} \rightarrow{ }^{2} \mathrm{~A}$ transition between 580 and $620 \mathrm{~nm}$. As the pulsed OPO produced wide-band excitation $(\sim 10 \mathrm{~nm})$, the precise wavelength where the contrast changes could not be resolved, but it must occur around $600 \mathrm{~nm}$. This agrees well with a previously reported PLE band assigned to $\mathrm{SiV}^{-}$, which commences at $605 \mathrm{~nm}$ (2.05 eV) [19]. The PLE band was previously concluded to be excitation into the diamond conduction band because of the absence of a ZPL and broad similarities with the diamond ultraviolet absorption band [19]. However, this was disputed by subsequent $a b$ initio calculations, which place the electronic levels of $\mathrm{SiV}^{-}$much deeper within the diamond band gap [15].

Here, we assign that PLE band to an absorption transition from the ground ${ }^{2} \mathrm{E}$ level to a ${ }^{2} \mathrm{~A}$ level $2.05 \mathrm{eV}$ above. We propose that the observed absence of a ZPL implies that this is a transition between electronic levels of the same parity $\left({ }^{2} E_{g}\right.$ and ${ }^{2} \mathrm{~A}_{1 \mathrm{~g}}$ ). Selection rules forbid purely electronic transitions between levels of the same parity, but they do allow transitions that involve the creation/annihilation of a phonon mode with odd parity [30]. This explains why a large PLE band can occur with no ZPL, but it requires inversion symmetry. This adds experimental support for the conventional split-vacancy $D_{3 d}$ model of $\mathrm{SiV}^{-}$.

It is known that other color centers in diamond have significant phonon sidebands which accompany transitions between electronic configurations that excite (quasi)local phonon modes of A symmetry [31]. As the $\mathrm{D}_{3 \mathrm{~d}}$ split-vacancy structure permits odd parity $A_{2 u}$ local displacements of the silicon atom and its six nearest-neighbor carbon atoms, it is very plausible that an $A_{2 u}$ vibrationally allowed ${ }^{2} E_{g} \rightarrow{ }^{2} A_{1 g}$ transition gives rise to the 605-nm PLE band. Given the small energy gap $\sim 0.37 \mathrm{eV}$ between the excited ${ }^{2} \mathrm{E}_{\mathrm{u}}$ and ${ }^{2} \mathrm{~A}_{1 \mathrm{~g}}$ levels, it is also plausible that rapid nonradiative ${ }^{2} \mathrm{~A}_{1 \mathrm{~g}} \rightarrow{ }^{2} \mathrm{E}_{\mathrm{u}}$ decay via a few phonons gives rise to the ${ }^{2} \mathrm{E}_{\mathrm{u}} \rightarrow{ }^{2} \mathrm{E}_{\mathrm{g}} \mathrm{PL}$ when the ${ }^{2} \mathrm{E}_{\mathrm{g}} \rightarrow{ }^{2} \mathrm{~A}_{1 \mathrm{~g}}$ transition is excited [32].

\section{POLARIZATION OF PHONON SIDEBAND}

Figure 7(b) demonstrates that the PLE polarization varies considerably between excitation wavelengths 690 and $737 \mathrm{~nm}$, which is within the absorption sideband of the ${ }^{2} E_{g} \rightarrow{ }^{2} E_{u}$ transition. The need for arbitrary laser wavelengths makes it challenging to examine this absorption sideband in PLE. Instead, the emission sideband was investigated using PL measurements with 532-nm excitation. We measured the PL polarization contrast within the emission phonon sideband at $8 \mathrm{~K}$ and at room temperature (RT), as shown in Fig. 8(a). The features of this sideband have been observed before $[19,28,33,34]$, so the discussion here will be restricted to their polarization.

The first sideband peak (at $41 \mathrm{meV}$ ) is entirely due to $d_{\perp}$. The sharp feature at $64 \mathrm{meV}$ is dominated by $d_{\|}$, like the ZPL [Fig. 8(b)], which is consistent with it being a local phonon mode of A symmetry [28]. The higher energy phonon peaks also have similar polarization dependence to the ZPL, but with lower contrast. At RT the phonon sideband peaks are less distinct, but roughly the same polarization characteristics are observed. The 41-meV peak disappears as it merges with the shoulders of the ZPL and the 64-meV peak, but its opposite

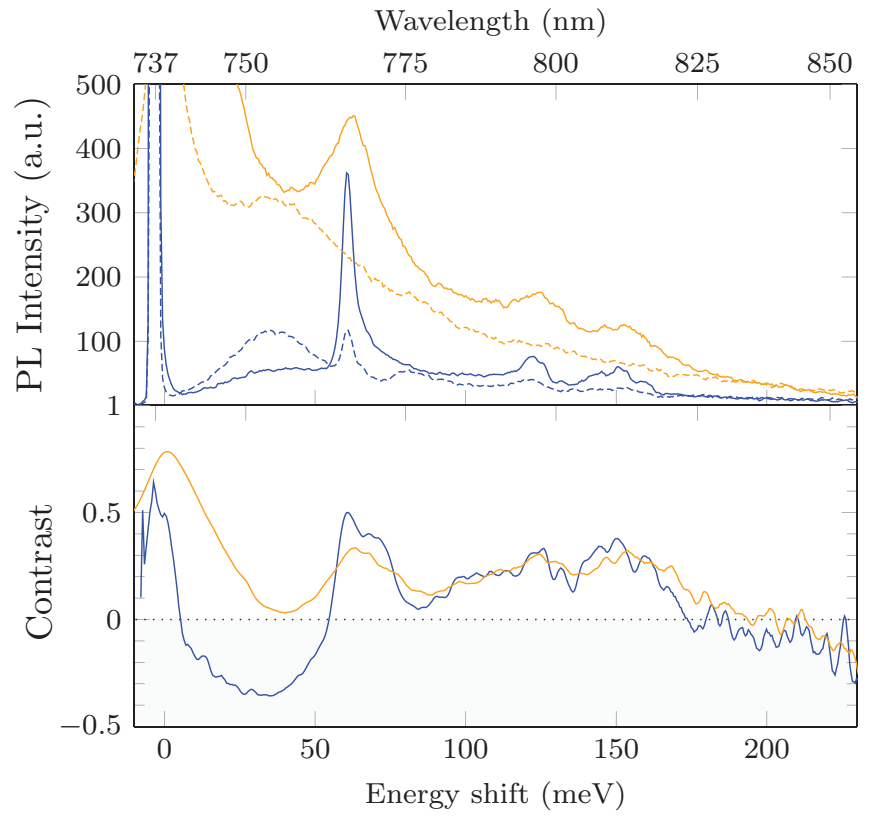

FIG. 8. (Color online) Polarization of the PL sideband. (a) PL sideband at $8 \mathrm{~K}$ (blue) and room temperature (orange) measured in the $\mathrm{XZ}$ direction (solid) and the $\mathrm{Y}$ direction (dashed). There is considerable variation in polarization, with the $41-\mathrm{meV}$ peak being polarized oppositely to the ZPL. (b) The contrast $C$ for $8 \mathrm{~K}$ (blue) and room temperature (orange). The ZPL shows greater contrast at RT.

polarization produces a small spectral region with $C=0 \%$. Interestingly, the ZPL contrast increases to $C=80 \%$ at $\mathrm{RT}$, which is of interest to RT applications using the center as a single-photon source.

The variation of optical polarization within the phonon sidebands of the ${ }^{2} \mathrm{E}_{\mathrm{g}} \rightarrow{ }^{2} \mathrm{E}_{\mathrm{u}}$ transition is strong evidence of dynamic Jahn-Teller effects within the ${ }^{2}$ E levels $[35,36]$.

\section{COMPARISON WITH PREVIOUS REPORTS $\mathrm{OF} \mathrm{SiV}^{-}$ALIGNMENT}

In this work, it has been established that the $\mathrm{SiV}^{-}$center is aligned along the $\langle 111\rangle$ crystal vectors. This conclusion agrees with previous ab initio calculations [15] and related EPR measurements of $\mathrm{SiV}^{0}$ [16], and is further confirmed by recent Zeeman measurements on low strain $\mathrm{SiV}^{-}$samples [18]. However, our result differs from previous optical polarization $[13,14]$ and Zeeman [17] studies. In this section, we discuss these previous studies in further detail.

Brown and Rand [13] concluded a $\langle 110\rangle$ alignment with a single dipole along $\mathrm{Z}$. They measured $\mathrm{SiV}^{-}$ensembles in $\mathrm{CVD}$ films, and the interpretation relied on assuming individual sites were randomly oriented along equivalent crystal vectors. We have seen evidence for preferential alignment of $\mathrm{SiV}^{-}$ sites incorporated during growth, and if similar effects were present in their CVD films then it is difficult to re-interpret the ensemble data. A reinterpretation is also complicated since they used 488-nm excitation, which we have shown to probe the $\mathrm{E}_{\mathrm{g}} \leftrightarrow \mathrm{A}_{1 \mathrm{~g}}$ transition. 
Neu et al. [14] made measurements on diamond nanoislands with $\{100\}$ surfaces, and deliberately avoided the $2.05-\mathrm{eV}$ excitation band. They saw two main sets of orientations, aligned with the $\langle 110\rangle$ nanoisland edges and separated by $90^{\circ}$ (their Fig. 7). When viewing into a $\{100\}$ surface the $\langle 111\rangle$ vectors are also aligned with $\langle 110\rangle$ crystal edges, and so their results are in fact consistent with $\mathrm{SiV}^{-}$having a $\langle 111\rangle$ orientation. In addition, they saw some $\mathrm{SiV}^{-}$sites angled at $45^{\circ}$ to the $\langle 110\rangle$ nanoisland edges (their Fig. 9), and attributed them to $\langle 110\rangle$ oriented sites. Still more sites were observed at seemingly arbitrary angles, however, suggesting that it is difficult to identify the precise crystal alignment of every nanoisland.

Their data indicate nearly $100 \%$ contrast, which is significantly higher than any measurement included here in Figs. 2 and 3. It is plausible that effects in the nanodiamond environment alter the apparent dipole moments, and measured contrast is dependent on background correction techniques. Additionally, we have shown that emission polarization varies significantly across the sideband, and does reach high contrast on the ZPL at room temperature. It is difficult to compare previous reports without knowing the spectral band over which emission was measured.

A $\langle 100\rangle$ orientation was tentatively concluded from Zeeman splitting results [17]. Zeeman measurements probe spin sublevels, which have not been addressed here. More recent and thorough Zeeman measurements have been subsequently reported that are compatible with a $\langle 111\rangle$ orientation [18].

\section{CONCLUSION}

We have established the $\mathrm{SiV}^{-}$to be aligned with the $\langle 111\rangle$ crystal vectors, having its strongest dipole moment along this symmetry axis. Our results are consistent with the defect site having $\mathrm{D}_{3 \mathrm{~d}}$ symmetry and a primary optical transition between two $\mathrm{E}$ states. Both of these $\mathrm{E}$ states are split at zero field by spin-orbit coupling. An additional higher ${ }^{2} \mathrm{~A}_{1 \mathrm{~g}}$ level allows efficient off-resonant excitation in a polarization orthogonal to the ZPL emission. At room temperature the ZPL polarization contrast increases from $C=60 \%$ to $80 \%$ and the ZPL approximates emission from a single dipole. These properties are of particular interest for applications such as cavity QED and quantum entanglement algorithms that require well polarized single photons.

\section{ACKNOWLEDGMENTS}

The authors acknowledge funding from ARC (DP120102232), EU (DIAMANT), ERC, German Science Foundation - DFG (SFB TR21, FOR1482, FOR1493), German Israeli Foundation, JST, DARPA, Sino-German Center and the Alexander von Humboldt foundation.
[1] C. Kurtsiefer, S. Mayer, P. Zarda, and H. Weinfurter, Phys. Rev. Lett. 85, 290 (2000).

[2] R. Brouri, A. Beveratos, J.-P. Poizat, and P. Grangier, Opt. Lett. 25, 1294 (2000).

[3] L. Childress, J. M. Taylor, A. S. Sørensen, and M. D. Lukin, Phys. Rev. Lett. 96, 070504 (2006).

[4] C.-C. Fu, H.-Y. Lee, K. Chen, T.-S. Lim, H.-Y. Wu, P.-K. Lin, P.-K. Wei, P.-H. Tsao, H.-C. Chang, and W. Fann, Proc. Natl. Acad. Sci. 104, 727 (2007).

[5] V. Vaijayanthimala and H.-C. Chang, Nanomedicine 4, 47 (2009).

[6] A. Ermakova, G. Pramanik, J.-M. Cai, G. Algara-Siller, U. Kaiser, T. Weil, Y.-K. Tzeng, H. C. Chang, L. P. McGuinness, M. B. Plenio, B. Naydenov, and F. Jelezko, Nano Lett. 13, 3305 (2013).

[7] M. W. Doherty, N. B. Manson, P. Delaney, F. Jelezko, J. Wrachtrup, and L. C. Hollenberg, Phys. Rep. 528, 1 (2013).

[8] A. T. Collins, L. Allers, C. J. Wort, and G. A. Scarsbrook, Diamond Relat. Mater. 3, 932 (1994).

[9] S. Benjamin, B. Lovett, and J. Smith, Laser Photonics Rev. 3, 556 (2009).

[10] E. Neu, C. Hepp, M. Hauschild, S. Gsell, M. Fischer, H. Sternschulte, D. Steinmüller-Nethl, M. Schreck, and C. Becher, New J. Phys. 15, 043005 (2013).

[11] C. Wang, C. Kurtsiefer, H. Weinfurter, and B. Burchard, J. Phys. B 39, 37 (2006).

[12] E. Neu, D. Steinmetz, J. Riedrich-Möller, S. Gsell, M. Fischer, M. Schreck, and C. Becher, New J. Phys. 13, 025012 (2011).

[13] S. W. Brown and S. C. Rand, J. Appl. Phys. 78, 4069 (1995).
[14] E. Neu, M. Fischer, S. Gsell, M. Schreck, and C. Becher, Phys. Rev. B 84, 205211 (2011).

[15] J. P. Goss, R. Jones, S. J. Breuer, P. R. Briddon, and S. Öberg, Phys. Rev. Lett. 77, 3041 (1996).

[16] U. F. S. D’Haenens-Johansson, A. M. Edmonds, B. L. Green, M. E. Newton, G. Davies, P. M. Martineau, R. U. A. Khan, and D. J. Twitchen, Phys. Rev. B 84, 245208 (2011).

[17] H. Sternschulte, K. Thonke, J. Gerster, W. Limmer, R. Sauer, J. Spitzer, and P. Münzinger, Diamond Relat. Mater. 4, 1189 (1995).

[18] C. Hepp, T. Müller, V. Waselowski, J. N. Becker, B. Pingault, H. Sternschulte, D. Steinmüller-Nethl, A. Gali, J. R. Maze, M. Atatüre, and C. Becher, Phys. Rev. Lett. 112, 036405 (2014).

[19] K. Iakoubovskii and G. J. Adriaenssens, Phys. Rev. B 61, 10174 (2000).

[20] T. Teraji, T. Taniguchi, S. Koizumi, K. Watanabe, M. Liao, Y. Koide, and J. Isoya, Jpn. J. Appl. Phys. 51, 090104 (2012).

[21] C. D. Clark and C. A. Norris, J. Phys. C 4, 2223 (1971).

[22] A. M. Edmonds, U. F. S. D’Haenens-Johansson, R. J. Cruddace, M. E. Newton, K.-M. C. Fu, C. Santori, R. G. Beausoleil, D. J. Twitchen, and M. L. Markham, Phys. Rev. B 86, 035201 (2012).

[23] A. T. Collins, J. Phys.: Condens. Matter 1, 439 (1989).

[24] K. Iakoubovskii, Phys. Rev. B 70, 205211 (2004).

[25] K. Iakoubovskii and A. T. Collins, J. Phys.: Condens. Matter 16, 6897 (2004).

[26] U. F. S. D’Haenens-Johansson, A. M. Edmonds, M. E. Newton, J. P. Goss, P. R. Briddon, J. M. Baker, P. M. Martineau, R. U. A. Khan, D. J. Twitchen, and S. D. Williams, Phys. Rev. B 82, 155205 (2010). 
[27] M. Tinkham, Group Theory and Quantum Mechanics (Dover, New York, 1964).

[28] H. Sternschulte, K. Thonke, R. Sauer, P. C. Münzinger, and P. Michler, Phys. Rev. B 50, 14554 (1994).

[29] C. D. Clark, H. Kanda, I. Kiflawi, and G. Sittas, Phys. Rev. B 51, 16681 (1995).

[30] A. M. Stoneham, Theory of Defects in Solids: Electronic Structure of Defects in Insulators and Semiconductors (Oxford University Press, New York, 2001).
[31] G. Davies, Rep. Prog. Phys. 44, 787 (1981).

[32] L. Rogers, Phys. Proc. 3, 1557 (2010).

[33] C. Clark and C. Dickerson, Surf. Coat. Technol. 47, 336 (1991). [34] T. Feng and B. D. Schwartz, J. Appl. Phys. 73, 1415 (1993).

[35] G. Davies and M. F. Hamer, Proc. R. Soc. London, Ser. A 348, 285 (1976).

[36] Kai-Mei C. Fu, C. Santori, P. E. Barclay, L. J. Rogers, N. B. Manson, and R. G. Beausoleil, Phys. Rev. Lett. 103, 256404 (2009). 